\title{
Teil A: Trägerschaft von BMB und geeignete Rechtsformen
}

A.1. Einführung

A.2. Welche Rechtsformen einer BMB sind juristisch möglich?

A.3. Welche Vor- und Nachteile haben denkbare Rechtsformen einer BMB?

A.4. Welcher spezifische Regelungsbedarf für den Aufbau und Betrieb einer BMB besteht in Abhängigkeit von der gewählten Rechtsform? 



\section{A.1. Einführung}

Als „Rechtsform“ eines Unternehmens bezeichnet man die rechtliche Verfassung, durch die eine Unternehmung ihre Rechtsbeziehungen im Innen- und Außenverhältnis regelt. Unternehmensformen können sowohl öffentlich-rechtlicher als auch privatrechtlicher Natur sein. Soweit aus der TMF-Befragung ${ }^{1} \mathrm{im}$ Teilprojekt o (TPo) von 2004 und weiteren Voruntersuchungen ${ }^{2}$ ersichtlich wird, überwiegen im nationalen und europäischen Bereich Biomaterialbanken (BMB) in öffentlich-rechtlicher Trägerschaft, die hauptsächlich von Kliniken oder universitären Einrichtungen unterhalten werden. Vereinzelte BMB in privater Trägerschaft firmieren entweder als eingetragener Verein (Brainnet e.V., HIV Bochum e.V. i.Gr.) oder als GmbH (WTZE).

Das TPo ergab zudem, dass drei der sechs befragten BMB bereits die öffentlich-rechtliche gegen eine private Trägerschaft eingetauscht hatten, und dass bei den verbleibenden drei BMB hieran zumindest ein starkes Interesse bestand. Die Tendenz zur Privatisierung wurde in der Befragung damit begründet, dass ein privater Träger womöglich schneller und effektiver arbeiten könne, als ein öffentlich-rechtlicher. In einem Fall wurde Bezug darauf genommen, dass ein Antrag auf Qualität sichernde Schritte in einer Anstalt des öffentlichen Rechts circa ein Jahr lang nicht bearbeitet wurde, die öffentlich-rechtliche Organisationsform also sehr langsam sei. Von derselben BMB wurde gleichzeitig aber betont, dass es bis dato keine Vorstellungen hinsichtlich alternativer Rechtsformen gäbe. Auch bei zwei anderen BMB in öffentlich-rechtlicher Trägerschaft gab es zwar noch keine konkreten Überlegungen zur Änderung der Rechtsform, es wurde aber ausdrücklich die Ansicht geäußert, dass ein Übergang in eine Stiftung oder eine andere privatrechtliche Form denkbar sei.

Allein die Tatsache, dass die Hälfte der von TPo untersuchten BMB schon in eine private Rechtsform übergegangen sind, rechtfertigt die Frage, welche Rechtsform für eine BMB die geeignete sein könnte, zumal die Wahl der Rechtsform auch Konsequenzen für die optimale praktische und organisatorische Ausgestaltung einer BMB haben dürfte. Im vorliegenden Gutachten soll es jedoch nicht darum gehen, die ,reale BMB-Welt mit den dort anzutreffenden (zugegebenermaßen unzureichenden) rechtlichen Organisationsformen “3 ausführlich darzustellen oder öffentlich-rechtliche Organisationsformen nochmals eingehend zu beleuchten. Stattdessen werden im Folgenden Alternativen einer privaten Trägerschaft von BMB aufgezeigt und die entsprechenden Rechtsformen hinsichtlich ihrer praktischen Eignung untersucht. Es ist in diesem Zusammenhang anzumerken, dass es der öffentlichen Hand unbenommen ist,

1 Goebel/Ihle (2004), TMF-BMB Befragung, Bericht und Materialsammlung Band 2 (zitiert: TP0), Mai 2004.

2 Vgl. z.B. Hirtzlin/Simon et al. (2003), An empirical survey on biobanking of human genetic material and data in six EU countries, in: European Journal of Human Genetics, 11, S. 475-488. Auch für die drei bekanntesten ausländischen BMB, nämlich die in Island, Estland und Großbritannien, ist und war die staatliche Organisation, Beteiligung und Kontrolle von großer Bedeutung (in Estland z.B. über eine Stiftung), selbst wenn die letztendliche Rechtsform privatrechtlich ist (Island und Estland). Bei diesen drei BMB handelt es sich allerdings um sehr groß angelegte Biomaterial- und Datensammlungen, verglichen mit den relativ kleinen BMB wie sie in Deutschland oder anderen europäischen Ländern zu finden sind. 
in bestimmten Fällen selbst wie ein privates Unternehmen aufzutreten oder sich an privaten Unternehmungen in beliebiger Höhe zu beteiligen. Da die öffentliche (Teil-)Trägerschaft in diesen Fällen allerdings nicht immer zu erkennen ist, obliegt es der öffentlichen Hand, gegebenenfalls Art und Umfang ihrer Beteiligung aus Reputationsgründen bei der Firmierung kenntlich zu machen.

\section{A.2. Welche Rechtsformen einer BMB sind juristisch möglich?}

\section{A.2.1. Kriterien und Merkmale}

Die Entscheidung über die Rechtsform einer Unternehmung hängt außer von deren Zielsetzungen unter anderem auch von der Betriebsgröße, der Möglichkeit der Kapitalbeschaffung, den gewünschten Haftungsregelungen und von steuerlichen Überlegungen ab. Deshalb bietet die Rechtsordnung der Bundesrepublik Deutschland mehrere Möglichkeiten für die Gestaltung einer Unternehmensverfassung. Bei der Einzelunternehmung wird das Eigenkapital von einer einzelnen Person, dem Unternehmer oder Inhaber, aufgebracht. In der Regel sind der Umfang dieser Mittel und damit die Betriebsgröße begrenzt. Eine Gesellschaftsunternehmung wird durch vertraglichen Zusammenschluss von zwei oder mehr Personen zur Verfolgung eines gemeinsamen Zweckes gegründet. Die Gründe für die Bildung einer Gesellschaft können mannigfacher Art sein:

- Erhöhung des Eigenkapitals

- Ergänzung der Arbeitskraft und Verteilung der Arbeitslast

- Verteilung oder Begrenzung des Risikos auf das Gesellschaftsvermögen

- Erhöhung der Kreditwürdigkeit durch Erweiterung der Haftung

- steuerliche Vorteile ${ }^{4}$

Im Falle einer BMB treten weitere Gründe hinzu, die gemeinsam mit obigen Aspekten zu den folgenden, hier angelegten Auswahlkriterien für die geeignete Rechtsform einer BMB geführt haben:

- Langwierige Entscheidungsprozesse sollten vermieden werden.

- Die BMB sollte eine auf Vertrauen basierende Akzeptanz in der Öffentlichkeit genießen.

- Der mit einer möglichen Insolvenz der BMB verbundene Fall des Untergangs oder der Fehlnutzung von Proben sollte vermieden werden.

- Die Trägerschaft sollte eine gewisse Garantie für die Nachhaltigkeit der BMB gewähren.

- Die BMB sollte finanziell weitestgehend unabhängig sein.

4 Kapitalgesellschaften sind mit ihrem Einkommen und Vermögen jeweils selbständig im Rahmen der Körperschafts- und Vermögenssteuer steuerpflichtig. Diese Doppelbelastung bei der Einkommensteuer wurde dadurch beseitigt, dass die auf die Ausschüttung entfallende Körperschaftsteuer auf die persönlichen Einkommen- bzw. Körperschaftsteuer angerechnet wurde. Das körperschaftssteuerliche Anrechnungsverfahren ist seit dem 1.1.2001 entfallen. Die Körperschaftsteuer liegt als Definitivsteuer nun bei $25 \%$, und zum Ausgleich werden die bezogenen Gewinnanteile als so genannte „Halbeinkünfteverfahren“ bei natürlichen Personen nur zur Hälfte angesetzt. 
- Der Übergang der BMB von einer Rechtsform in eine andere sollte problemlos möglich sein.

- Die beteiligten Forscher sollten von etwaiger Haftung weitestgehend ausgeschlossen sein.

- Die Wirtschaftlichkeit einer BMB diente hier zunächst nicht als Kriterium, könnte aber für zukünftige Entscheidungen eine zentrale Rolle spielen.

Je nachdem, ob die Gesellschafter den Gläubigern gegenüber persönlich haften oder nur das Gesellschaftsvermögen der juristischen Person, unterscheidet man Personen- und Kapitalgesellschaften. Haftung bedeutet in diesem Zusammenhang, dass die Gesellschafter oder die Gesellschaft Außenstehenden gegenüber für die Verbindlichkeiten der Gesellschaft mit ihrem Vermögen einstehen müssen. Im Fall der BMB erscheinen Rechtsformen, die keine Haftungsbeschränkung der Personengesellschafter bzw. Inhaber erlauben oder unbeschränkt haftende Elemente beinhalten, von vornherein als ungeeignet. Ebenso scheiden alle Rechtsformen aus, die für die Zusammenarbeit freiberuflicher Berufsträger zur Erbringung personal geprägter Dienste gedacht sind. Demzufolge kommen Personengesellschaften für die Rechtsform einer BMB nicht in Betracht und werden hier ebenso wenig behandelt wie freiberufliche Berufsträger und die für sie bzw. ihre Zusammenarbeit geschaffenen Rechtsformen.

\section{A.2.2. Mögliche Rechtsformen}

Nachfolgend werden die wichtigsten, für den Betrieb einer BMB in Frage kommenden Rechtsformen kursorisch dargestellt. Auf steuerrechtliche Aspekte soll wegen der damit verbundenen besonderen Schwierigkeiten nicht eingegangen werden. Hierzu sei für jeden Einzelfall eine Beratung und dauerhafte Begleitung durch steuerrechtliche Experten anempfohlen.

\section{A.2.2.1. Die Gesellschaft bürgerlichen Rechts (GbR)}

\begin{tabular}{|l|l|}
\hline Firma & $\begin{array}{l}\text { Name mindestens eines Gesellschafters mit dem Zusatz „GbR“; } \\
\text { kann durch Zweckbezeichnung ergänzt werden }\end{array}$ \\
\hline Gesellschaftsrechtliche Regelung & $\$ \$ 705$ ff. BGB \\
\hline Gesellschafter & mindestens zwei Inhaber \\
\hline Geschäftsführung & $\begin{array}{l}\text { alle Gesellschafter gemeinsam, es sei denn, der Gesellschafts- } \\
\text { vertrag schließt einzelne Gesellschafter von der Geschäftsfüh- } \\
\text { rung aus }\end{array}$ \\
\hline Eigenkapitalbeschaffung & Einlage der Inhaber aus dem Privatvermögen. \\
\hline
\end{tabular}




\begin{tabular}{|l|l|l|l|}
\hline Haftung & $\begin{array}{l}\text { Jeder Inhaber haftet grundsätzlich mit seinem gesamten } \\
\text { Vermögen für die Verbindlichkeiten des Unternehmens. Ein } \\
\text { Gläubiger kann nach neuester BGH-Rechtsprechung einzelne } \\
\text { Gesellschafter oder die Gesellschaft als Ganzes in Anspruch } \\
\text { nehmen. }\end{array}$ \\
\hline Gewinnverteilung & $\begin{array}{l}\text { Der Gewinn steht den Gesellschaftern grundsätzlich anteilig } \\
\text { gemäß Gesellschaftsvertrag zu. }\end{array}$ \\
\hline Steuern & Umsatz-, Einkommen-, evtl. Gewerbesteuer \\
\hline
\end{tabular}

Vorteile: Vor allem geeignet für kleine Betriebe oder Familienunternehmen bzw. bei engem Vertrauensverhältnis zwischen den Inhabern.

Nachteile: Meinungsverschiedenheiten zwischen den Gesellschaftern können den Geschäftsablauf lähmen; volle Haftung der Gesellschafter. Grundsätzlich ist nach \7I7 S. 2 BGB die Gesellschafterstellung im Zweifel nicht übertragbar. Ein Wechsel der Mitgliedschaft ist aber dann problemlos möglich, wenn der Gesellschaftsvertrag dies zulässt oder alle Gesellschafter zustimmen und die Mitgliedschaft mit dem Anteil am Gesellschaftsvermögen zusammen übertragen wird.

\section{A.2.2.2. Die Gesellschaft mit beschränkter Haftung (GmbH)}

\begin{tabular}{|l|l|}
\hline Firma & $\begin{array}{l}\text { Sachbezeichnung mit dem Zusatz „GmbH“; auch Sach-, } \\
\text { Phantasie- und Mischfirmen sind zulässig. }\end{array}$ \\
\hline Gesellschaftsrechtliche Regelung & GmbHG \\
\hline Gesellschafter & mindestens ein Gründer („Ein-Personen-GmbH“) \\
\hline Geschäftsführung & $\begin{array}{l}\text { Geschäftsführer, Gesellschafterversammlung (bei mehr als 500 } \\
\text { Arbeitnehmern zusätzlich ein Aufsichtsrat) }\end{array}$ \\
\hline Eigenkapitalbeschaffung & $\begin{array}{l}\text { Stammeinlagen der Gesellschafter in Höhe von derzeit } \\
\text { mindestens 25.000 € }\end{array}$ \\
\hline Haftung & $\begin{array}{l}\text { Für die Verbindlichkeiten der GmbH haftet nur das Vermögen } \\
\text { der Gesellschaft. Kreditgeber lassen sich aber vielfach die } \\
\text { Haftung mit dem Privatvermögen der Gesellschafter zusichern } \\
\text { und höhlen auf diese Weise die Grundidee der GmbH aus. }\end{array}$ \\
\hline Gewinnverteilung & $\begin{array}{l}\text { an die Gesellschafter entsprechend dem Anteil ihrer Stammein- } \\
\text { lagen }\end{array}$ \\
\hline Steuern & \begin{tabular}{l} 
Umsatz-, Gewerbe-; Körperschaftssteuer \\
\hline
\end{tabular}
\end{tabular}

Vorteile: Eine GmbH kann auch für nicht-gewerbliche wie z.B. wissenschaftliche Zwecke gegründet werden. Sie ist ebenso für Familienunternehmen wie für öffentliche Träger geeignet. Im Bereich der wissenschaftlichen Forschung hat 
sie sich bereits als geeignete Rechtsform bewährt. Die $\mathrm{GmbH}$ eignet sich besonders zur Ausgliederung bestimmter Funktionen aus einer Unternehmung und für den Zusammenschluss von Unternehmungen zur Verfolgung bestimmter Zwecke. Ihre Gründung ist mit wenig Kapital möglich (derzeit $25.000 €)$. Die Nennung von Inhaber- oder Gesellschafternamen ist nicht zwingend. Die Haftung einer GmbH ist, wie der Name sagt, beschränkt. Bei der Ein-Personen-GmbH hat der „Gesellschafter" die gleiche volle Handlungsfreiheit wie ein Einzelunternehmer, muss jedoch nicht wie dieser persönlich haften. Durch das Organ der Gesellschafterversammlung besteht ein weitgehendes Mitverwaltungsrecht der Gesellschafter. Die Gründungs- und Verwaltungskosten sind bei der $\mathrm{GmbH}$ niedriger als bei einer Aktiengesellschaft. Als juristische Person ist die Fortführung des Unternehmens auch im Falle des Ausscheidens oder Auswechselns von Gesellschaftern grundsätzlich gewährleistet. Geschäftsanteile an einer $\mathrm{GmbH}$ sind veräußerlich und vererblich ( $\mathbb{1}$ I5 Abs. I GmbHG). In der Regel werden hierfür jedoch im Statut der GmbH Genehmigungsvorbehalte der übrigen Gesellschafter aufgestellt.

Nachteile: Meinungsverschiedenheiten zwischen den Gesellschaftern können sich negativ auswirken. In der Gründungsphase und ohne überobligationsmäßiges Stammkapital können sich Kapitalbeschaffungsprobleme bei Banken ergeben. Bei Mindeststammkapital erfolgt regelmäßig keine Kreditvergabe an die $\mathrm{GmbH}$ ohne persönliche Bürgschaft und/oder Gestellung sonstiger Sicherheiten seitens der Gesellschafter.

\section{A.2.2.3. Die Aktiengesellschaft (AG)}

\begin{tabular}{|l|l|}
\hline Firma & $\begin{array}{l}\text { Sachbezeichnung mit dem Zusatz „Aktiengesellschaft" oder } \\
\text { „AG“. Es sind auch Sach-, Phantasie- und Mischfirmen zulässig. }\end{array}$ \\
\hline Gesellschaftsrechtliche Regelung & AktG \\
\hline Gesellschafter & Einmann-Gründung ist möglich. \\
\hline Geschäftsführung & $\begin{array}{l}\text { Vorstand (Führung der laufenden Geschäfte), Aufsichtsrat } \\
\text { (Überwachung des Vorstands), Hauptversammlung der } \\
\text { Aktionäre }\end{array}$ \\
\hline Eigenkapitalbeschaffung & $\begin{array}{l}\text { Die AG gibt Aktien gegen Einlagenzahlung an ihre Aktionäre aus. } \\
\text { Der Gesamtnennwert aller umlaufenden Aktien muss } \\
\text { mindestens 50.000 } € \text { betragen. }\end{array}$ \\
\hline Haftung & \begin{tabular}{l} 
Für Verbindlichkeiten haftet nur das Gesellschaftsvermögen. \\
\hline Gewinnverteilung
\end{tabular} \\
\hline $\begin{array}{l}\text { In der Regel steht den Aktionären entsprechend ihrem } \\
\text { nominellen Aktienanteil am Grundkapital ein Anteil am } \\
\text { ausgeschütteten lahresüberschuss der Gesellschaft zu. }\end{array}$ \\
\hline Steuern & $\begin{array}{l}\text { Umsatz-, Körperschafts-, Vermögenssteuer; Einkommenssteuer } \\
\text { bei den Aktionären. }\end{array}$ \\
\hline
\end{tabular}


Vorteile: Keine persönliche Haftung. Ein Aktionär kann sich kapitalmäßig bei beschränktem Verlustrisiko beteiligen, ohne persönlich haften oder mitarbeiten zu müssen. Die AG ist besonders für große Unternehmen mit sehr hohem Kapitalbedarf geeignet. Die Übertragung von Gesellschaftsanteilen und damit die Einbeziehung neuer „Gesellschafter“ (Aktionäre) sind durch die Übernahme von Aktien in der Regel problemlos möglich.

Nachteile: Bei der Aktiengesellschaft handelt es sich grundsätzlich um eine sehr kapitalaufwendige und wegen einer Reihe von Bestimmungen zum Schutz der wirtschaftlichen Partner relativ aufwendig zu führende Unternehmensform. Es ist aber durchaus denkbar, dass die AG mit ihrer besonderen Kontrollkultur nach einer längeren Vorlaufzeit in einer anderen Rechtsform, z.B. als Verein, für den finalen Betrieb einer BMB gewählt werden könnte.

\section{A.2.2.4. Der eingetragene Verein (e.V.)}

\begin{tabular}{|c|c|}
\hline Name & Sachbezeichnung mit dem Zusatz „e.V.“ \\
\hline Gesellschaftsrechtliche Regelung & of $21 \mathrm{ff} . \mathrm{BGB}$ \\
\hline Gesellschafter & bei Gründung mindestens sieben \\
\hline Geschäftsführung & $\begin{array}{l}\text { Vorstand (Führung der laufenden Geschäfte), Mitgliederver- } \\
\text { sammlung }\end{array}$ \\
\hline Eigenkapitalbeschaffung & Mitgliedsbeiträge \\
\hline Haftung & $\begin{array}{l}\text { Für Verbindlichkeiten haftet grundsätzlich nur das Vereinsvermö- } \\
\text { gen. }{ }^{5}\end{array}$ \\
\hline Gewinnverteilung & $\begin{array}{l}\text { Der eintragungsfähige Verein ist nicht auf einen wirtschaftli- } \\
\text { chen Geschäftsbetrieb und damit auch nicht auf Gewinnerzie- } \\
\text { lung ausgerichtet. }\end{array}$ \\
\hline Steuern & $\begin{array}{l}\text { Körperschaftssteuer (wirtschaftliche Vereine auch Gewerbe- und } \\
\text { Umsatzsteuer). }\end{array}$ \\
\hline
\end{tabular}

Vorteile: Keine persönliche Haftung der Mitglieder. Die Mitglieder dürfen die Angebote und Einrichtungen des Vereins eventuell gegen Entrichtung einer benutzungsabhängigen Zuzahlung zum Mitgliedsbeitrag nützen. Der Verein hat sich als Rechtsform in der wissenschaftlichen Forschung bereits bewährt. Je nach Satzung, kann die Aufnahme neuer Mitglieder erleichtert oder erschwert werden.

Nachteile: Meinungsverschiedenheiten innerhalb des Vorstands und zwischen diesem und der Mitgliederversammlung können zur Beeinträchtigung des Geschäftsbetriebs führen. 


\section{A.2.2.5. Die eingetragene Genossenschaft (e.G.)}

\begin{tabular}{|l|l|}
\hline Firma & Zweckbezeichnung mit dem Zusatz „Genossenschaft“ oder „e.G.“ \\
\hline Gesellschaftsrechtliche Regelung & GenG und HGB \\
\hline Gesellschafter & mindestens sieben \\
\hline Geschäftsführung & $\begin{array}{l}\text { Vorstand (Führung der laufenden Geschäfte), Aufsichtsrat } \\
\text { (Überwachung des Vorstands), Generalversammlung der } \\
\text { Genossen }\end{array}$ \\
\hline Eigenkapitalbeschaffung & $\begin{array}{l}\text { Die Genossenschaft bietet Geschäftsanteile an, auf die eine } \\
\text { gewisse Mindesteinzahlung zu leisten ist. }\end{array}$ \\
\hline Haftung & $\begin{array}{l}\text { In der Regel haftet jeder Genosse in Höhe seiner durch die } \\
\text { Anzahl der Geschäftsanteile bestimmten Haftsumme für die } \\
\text { Verluste der Genossenschaft. Bei Genossenschaften mit } \\
\text { unbeschränkter Haftung haften die Genossen jedoch mit ihrem } \\
\text { gesamten Privatvermögen. }\end{array}$ \\
\hline Gewinnverteilung & $\begin{array}{l}\text { Gewinne der Genossenschaft werden dem Geschäftsguthaben } \\
\text { gutgeschrieben. Dessen anteiliger Umfang ergibt sich aus der } \\
\text { Anzahl der Genossenschaftsanteile. }\end{array}$ \\
\hline Steuern & \begin{tabular}{l} 
Umsatz-, Körperschafts-, Vermögenssteuer \\
\hline
\end{tabular}
\end{tabular}

Vorteile: Zumeist keine persönliche Haftung. Der Genosse kann sich kapitalmäßig bei beschränktem Verlustrisiko beteiligen, ohne persönlich haften oder mitarbeiten zu müssen. Er kann die Einrichtungen der Genossenschaft nutzen. Die Genossenschaft ist besonders zur Selbsthilfe einzelner, wirtschaftlich relativ Schwacher geeignet, um einheitlich am Markt oder im Wettbewerb aufzutreten. Diese Wirkung kann noch dadurch erhöht werden, dass sich einzelne Genossenschaften zu Verbänden zusammenschließen.

Nachteile: Meinungsverschiedenheiten bei den Selbstverwaltungsorganen zwischen den Mitgliedern, die unabhängig von der Höhe ihrer Geschäftsguthaben bei Abstimmungen und Beschlüssen gleichberechtigt sind.

\section{A.2.2.6. Die Stiftung des privaten Rechts}

\begin{tabular}{|l|l|}
\hline Firma & Namens- oder Zweckbezeichnung mit dem Zusatz „Stiftung“" \\
\hline Gesellschaftsrechtliche Regelung & $\$ \$ 80 \mathrm{ff.} B G \mathrm{~B}$ \\
\hline Gesellschafter & mindestens ein Stifter \\
\hline Geschäftsführung & $\begin{array}{l}\text { Vorstand; die Satzung kann die Bildung eines Stiftungsrats zur } \\
\text { Überwachung des Vorstands vorsehen. }\end{array}$ \\
\hline
\end{tabular}


Teil A: Trägerschaft von BMB und geeignete Rechtsformen

\begin{tabular}{|l|l|}
\hline Eigenkapitalbeschaffung & $\begin{array}{l}\text { Eine natürliche oder juristische Person (bei Stiftungen des } \\
\text { öffentlichen Rechts eine Gebietskörperschaft) stellt Vermögen } \\
\text { oder Vermögenswerte für bestimmte Zwecke zur Verfügung. }\end{array}$ \\
\hline Haftung & Es haftet das Stiftungsvermögen. \\
\hline Gewinnverteilung & $\begin{array}{l}\text { Erträge werden dem Stiftungsvermögen zugeführt bzw. zur } \\
\text { Verfolgung des Stiftungszwecks eingesetzt. }\end{array}$ \\
\hline Steuern & Körperschafts-, Vermögenssteuer \\
\hline
\end{tabular}

Vorteile: Keine persönliche Haftung. Bereits bewährt zur Unterstützung von Forschungsprojekten.

Nachteile: Meinungsverschiedenheiten bei den Selbstverwaltungsorganen hemmen den Geschäftsbetrieb. Nach Anerkennung der Stiftung bestehen keine Widerrufsmöglichkeiten. Das auf die Stiftung übertragene Vermögen verbleibt während des Bestehens der Stiftung als juristische Person selbständig bei dieser. Die Höhe des Stiftungsvermögens ist rechtlich nicht festgelegt. Es ist zwar konstitutives Merkmal der Stiftung, kann aber sogar zeitweise fehlen. In diesem Fall besteht allerdings die Gefahr, dass die Genehmigung zur Errichtung der Stiftung zurückgenommen wird. Nur die Gewinne der Stiftung unterliegen der Körperschaftssteuer, bei gemeinnützigen Stiftungen ist aber auch hiervon eine Befreiung möglich. Nach gängiger Praxis der Aufsichtsbehörden für das Stiftungswesen genehmigen diese Stiftungen in der Regel nur, wenn sie bei ihrer Gründung ein Mindestkapital von derzeit ca. 500.000 bis 1.000.000€ aufweisen. Geringere Beträge erbringen in einer Zeit niedriger Zinsen zu wenig Ertrag, um der Stiftung die für ihre Zweckerreichung notwendigen Mittel zu verschaffen.

\section{A.2.3. Änderung der Rechtsform}

Änderungen der einmal gewählten Rechtsform einer BMB sind grundsätzlich möglich und auf Grund von Veränderungen der Zielrichtung einer BMB unter Umständen auch erstrebenswert. Die Einwilligungen der Spender dürfen durch Rechtsformveränderungen jedoch nicht berührt werden. Unter Umständen muss also die Nutzung einzelner Proben dann ausgeschlossen werden, wenn eindeutig erkennbar ist, dass die Einwilligungserklärung oder der Eigentumsübertrag an eine bestimmte Rechtsform gebunden ist. Dieser Fall könnte z.B. eintreten, wenn der Wechsel vom gemeinnützigen e.V. zur GmbH oder einer anderen wirtschaftlich auf Gewinn ausgerichteten Gesellschaftsform den Interessen des Einwilligenden entgegenläuft, da dieser keine kommerzielle Nutzung seiner Probe wünschte oder die Entscheidungs- und Kontrollmechanismen in einem Verein besonders schätzte. Eine solche Bindung der Einwilligung an eine spezielle Rechtsform muss aber aus der Einwilligungserklärung eindeutig hervorgehen. Ansonsten gelten alle zwischen Spendern und BMB getroffenen Vereinbarungen auch bei einer Rechtsformveränderung weiter. Alle mit den Proben verbundenen Eigentums-, Persönlichkeits- und Daten- 
schutzrechte bleiben bestehen. Im Falle einer Verletzung dieser Rechte würde sich die BMB schadenersatzpflichtig machen, und auch strafrechtliche Konsequenzen wären im Falle einer Verletzung der Schweigepflicht denkbar.

Juristisch ist bei der Veränderung der Rechtsform zwischen Umgründung und Umwandlung zu unterscheiden. Bei der Umgründung handelt es sich zwar wie bei der Umwandlung um die Überführung eines Unternehmens aus einer Rechtsform in eine andere. Allerdings erfolgt erstere prinzipiell im Wege der Auflösung und Neugründung, während letztere ohne Liquidation im Wege der Gesamtrechtsnachfolge geschieht. Im Zusammenhang mit BMB dürfte lediglich die Umwandlung von Interesse sein, weil sie die Liquidation und den damit verbundenen, erheblichen finanziellen und organisatorischen Aufwand vermeidet. Beide Vorgänge, Umwandlung und Umgründung, finden ihre gesetzliche Regelung im Umwandlungsgesetz. Der nach Maßgabe dieses Gesetzes erfolgte Umwandlungsbeschluss eines Unternehmens ersetzt dessen Auflösung und Abwicklung. Weitere Umwandlungsbestimmungen sind in

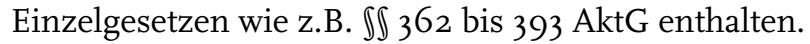

Die Umwandlung einer Unternehmung kann entweder formwechselnd oder übertragend erfolgen. Bei erstgenannter Variante wird nur die Rechtsform des Unternehmens geändert, ohne dass es zur Übertragung von Geschäfts- oder Gesellschaftsvermögen kommt. Ein Formwechsel könnte z.B. dann sinnvoll sein, wenn eine BMB sich mehr und mehr gewerblich orientiert und dieses Gewinnerwirtschaftungsziel ausdrücklich zum Gegenstand ihrer Rechtsform machen möchte, etwa in Form einer AG. Hierzu ist lediglich der Umwandlungsbeschluss der Gesellschafter der formwechselnden Gesellschaft erforderlich. Die wirtschaftliche Kontinuität bleibt gewahrt und der Rechtsträger tauscht nur die Rechtsform aus. ${ }^{6}$ Bei der zweiten Variante einer Umwandlung wird das Unternehmen aufgelöst und das Vermögen durch Verschmelzung oder Neuerrichtung auf eine andere Rechtspersönlichkeit übertragen. So könnten z.B. mehrere BMB beschließen, ihr Vermögen und ihren Bestand an Proben als Ganzes auf einen anderen Rechtsträger übertragen zu wollen. Die übertragenden Rechtsträger gehen im Zuge dieser Verschmelzung rechtlich unter, ohne dass erst eine Auflösung erfolgen muss. Auch eine Teilübertragung ist möglich, etwa um besondere Regelungen für die Probenbestände zu treffen.

\section{A.2.4. Zusammenfassung}

Die unter A.2. gemachten Ausführungen und das unter A.3.3. abgebildete Tableau können nur einen ersten Anhalt für die Entscheidung hinsichtlich der Rechtsform einer BMB bieten. Die jeweiligen konkreten Erfordernisse werden für die Wahl von entscheidender Bedeutung sein und die unter A.2.2.I. bis A.2.2.6. erläuterten Rechtsformen gewähren zudem in ihrer praktischen Ausgestaltung erheblichen Spielraum. Ein Wechsel der Rechtsform einer BMB ist durchaus möglich, um einer Änderung der Zielrichtung, z.B. von ideeller Forschung zu materieller Gewinnerwirtschaftung, formal Rechnung zu tragen. 
Teil A: Trägerschaft von BMB und geeignete Rechtsformen

Das deutsche Recht bietet dafür eine Vielzahl von Möglichkeiten. Für den Spender treten bei einem Formwechsel keine Änderungen in seinen Rechten ein, da grundsätzlich alle in der Patienteneinwilligung formulierten Vorgaben auch vom Rechtsnachfolger der BMB eingehalten werden müssen.

\section{A.3. Welche Vor- und Nachteile haben denkbare Rechtsformen einer BMB?}

Bei der Untersuchung der Vor- und Nachteile denkbarer Rechtsformen einer BMB wurden die folgenden, von TPo ausgearbeiteten Modellszenarien berücksichtigt:

- stark zentralistisches Modell,

- dezentrales Modell mit einer starken Zentraleinrichtung und

- dezentrales Modell mit kooperativer Struktur.

Alle drei Szenarien sind grundsätzlich sowohl in öffentlich-rechtlicher als auch in privatrechtlicher Trägerschaft denkbar.7 TPo hat jedoch nicht ohne Grund ergeben, dass die öffentlich-rechtliche Trägerschaft traditionell die typische Organisationsform des zentralistischen Modells ist. Die Ursache hierfür wird in erster Linie darin zu suchen sein, dass derartige Materialsammlungen vordringlich bei Universitäten bzw. Kliniken angesiedelt sind und dieser „historische“ Ursprung bestimmend für die Rechtsform der Materialbank war. Zudem gibt es im öffentlich-rechtlichen Bereich keine strukturelle Präformation für eine bestimmte Organisationsform, so dass dort alle drei Modellszenarien grundsätzlich möglich sind.

\section{A.3.1. Privatrechtliche Gestaltungen und Reduzierung der Formen}

Für die privatrechtliche Trägerschaft einer BMB haben sich einige Rechtsformen als besonders zweckmäßig herausgestellt, was in der folgenden Untersuchung und im anschließenden Rechtsformtableau (A.3.3.) näher erläutert wird. Dabei werden auch einige „weiche“ Kriterien angelegt, die als spezifisch für den Aufbau einer BMB ermittelt wurden.

\section{A.3.1.1. Die Gesellschaft bürgerlichen Rechts (GbR)}

Die Mitglieder einer GbR können jeweils selbst wieder Gesellschaften unterschiedlichen rechtlichen Zuschnitts sein. Insofern eignet sich die GbR besonders für einen dezentralen oder kooperativen BMB-Verbund mit mehr oder weniger starker Zentrale. Ziel der Gründung einer GbR ist nach den grundlegenden Bestimmungen der $\int \mathbb{\int} 705$ ff. BGB, dass sich die Gesellschafter zu

7 Die öffentlich-rechtliche Trägerschaft wird im Folgenden zwar implizit mitbehandelt, zentraler Gegenstand der Untersuchung sind aber die für die Praxis wesentlichen privatrechtlich organisierten Gesellschaftsmodelle. 
einem bestimmten Zweck zusammenfinden und dazu bestimmte Beiträge leisten. Diese Beiträge schließen auch die Erhebung, Speicherung und Weiterleitung oder Nutzung biologischen Materials ein. Die Beiträge der Gesellschafter können je nach Zweckrichtung unterschiedlicher Art sein und z.B. in finanziellen Beiträgen, Materialien oder der Wahrnehmung bestimmter Funktionen im Verbund bestehen.

Wie bei nahezu allen Gesellschaftsformen kann auch die innervertragliche Gestaltung einer GbR recht flexibel gehandhabt werden. Zwar ist grundsätzlich vorgesehen, dass alle Gesellschafter einer GbR auch Geschäftsführer sind, dies ist zur Vermeidung der schwerfälligen Handhabung einer Unternehmung aber durchaus auch anders gestaltbar. Grundsätzlich haftet jeder Inhaber mit seinem gesamten Vermögen für die Verbindlichkeiten des Unternehmens. Diese Vorschrift ist aber dann weniger problematisch, wenn es sich bei den Gesellschaftern selbst wieder um Unternehmen unterschiedlicher Rechtsform handelt. Deren Außenhaftung ist im Falle eines Durchgriffs entsprechend ihrer Gesellschaftsformen beschränkt, so dass z.B. eine beteiligte $\mathrm{GmbH}$ maximal mit ihrem Gesellschaftsvermögen haftet. Ein Durchgriff auf den dahinter stehenden einzelnen Gesellschafter kann in der Regel nicht vorgenommen werden. ${ }^{8}$

Wenn eine GbR über eine organisatorisch gut gestaltete Managementeinheit verfügt, können dadurch langwierige Entscheidungsprozesse vermieden werden. Hierfür gibt es eine Fülle von praktischen Beispielen. Der Zusammenschluss mehrerer BMB zu einem kooperativen Verbund würde zudem die öffentliche Akzeptanz der gesamten Institution stärken, zumal das dezentrale Modell ohnehin ein Funktionsverband ist, dem Fachvertrauen entgegengebracht würde. Wenn es sich bei den Gesellschaftern der GbR um öffentlichrechtliche Träger oder um Gesellschafter in kapitalgesellschaftsrechtlicher Gestalt handelt, spricht auch viel für die Langlebigkeit der BMB. Für den Fall der Insolvenz eines Gesellschafters könnte vereinbart werden, dass ein anderer Gesellschafter einspringt oder gemeinsam eine Lösung gefunden wird, die auf den Erhalt der Biomaterialien ausgerichtet ist. Die finanzielle Unabhängigkeit eines jeden Partners richtet sich gleichermaßen nach seinen eigenen finanziellen Verhältnissen und der Leistungsfähigkeit des Verbunds. Die Umwandlung eines dezentralen kooperativen Netzes in eine andere Gesellschaftsform ist relativ schwierig, weil alle Gesellschafter davon jeweils einzeln betroffen wären. Die Haftung des einzelnen Forschers richtet sich nach seiner Einbindung in die Gesellschaftsform, die an dem Verbund jeweils beteiligt ist.

\section{A.3.1.2. Die eingetragene Genossenschaft (e.G.)}

Die e.G. bezweckt nach \I GenG als Gesellschaft mit einer nicht geschlossenen Mitgliederzahl die Förderung des Erwerbes oder der Wirtschaft ihrer Mitglieder durch einen gemeinschaftlichen Geschäftsbetrieb. Die e.G. ist Inhaberin ihres

8 Es sei darauf hingewiesen, dass in die Finanzierung einer $\mathrm{GmbH}$ in der Regel auch die private Haftung der Gesellschafter einbezogen wird. In diesem Zusammenhang wird auch von einer Aushöhlung der Rechtsform der Gesellschaft mit „beschränkter“ Haftung gesprochen. 
Vermögens, wobei jedoch kein bestimmtes Grund- oder Stammkapital vorgegeben ist. Statt der Genossen haftet nur das Kapital der e.G. gegenüber den Gläubigern. Wenn sich die Genossen ein Statut mit Nachschusspflicht gegeben haben, kann sie aber im Falle der Insolvenz eine Haftungsverpflichtung treffen. Die fehlende Vorgabe eines festen Stamm- oder Grundkapitals gilt allgemein als Nachteil einer e.G. Wenn in schwierigen Zeiten die Erhöhung des Nennbetrags der Geschäftsanteile beschlossen werden muss, um neues Kapital zu erschließen, werden in der Regel viele Genossen austreten. In diesem Fall muss ihnen das Auseinandersetzungsguthaben ${ }^{9}$ ausgezahlt werden, so dass das Gegenteil der Kapitalerhöhung eintreten wird, nämlich eine Existenzgefährdung.

Ein grundsätzlicher Vorteil der e.G. ist deren regelmäßige Pflichtprüfung durch einen Prüfungsverband. Ab einer Bilanzsumme von 2.000.000 € muss eine solche Prüfung jährlich stattfinden ( $\mathbb{5} 53 \mathrm{ff}$. GenG). Sie erstreckt sich auf die Einrichtungen der e.G., ihre Vermögenslage und ihre Geschäftsführung einschließlich der Führung der Mitgliederliste. Die Prüfung dient der Feststellung der wirtschaftlichen Verhältnisse und der Ordnungsmäßigkeit der Geschäftsführung; sie ist allerdings mit erheblichen Kosten verbunden.

Im Kontext der BMB könnte sich die e.G. als Organisationsform für Patientenkollektive anbieten. Da für ihre Führung ein Vorstand zuständig ist, ließen sich langwierige Entscheidungsprozesse in der Regel vermeiden. Meinungsverschiedenheiten zwischen den Mitgliedern könnten sich aber lähmend auf die Arbeit der Selbstverwaltungsorgane auswirken, da alle Genossen unabhängig von der Höhe ihrer Geschäftsguthaben gleichberechtigt bei Abstimmungen und Beschlüssen sind. Genossenschaften genießen ein gewisses Vertrauen in der Öffentlichkeit, verfügen aber im deutschen Gesellschaftsleben über kein besonders gutes Image. Dennoch gab es schon I994 etwa 8000 Genossenschaften mit über I7 Millionen Mitgliedern und auch die wirtschaftliche Bedeutung (Volks- und Raiffeisenbanken) dieser Rechtsform ist erheblich. Die finanzielle Unabhängigkeit einer e.G. hängt von der Höhe der Einlagen der Genossen und der Anzahl ihrer Mitglieder ab. Das Umwandlungsbereinigungsgesetz (UmwBerG) von 1994 hat die Umwandlungsmöglichkeiten für Genossenschaften wesentlich erweitert. In Folge dessen können sie als formwechselnder Rechtsträger sowohl an einem Formwechsel beteiligt sein ( $\$ I9I Abs. I UmwBerG) oder Rechtsträger einer neuen Rechtsform sein ( $\int$ I9I Abs. 2 UmwBerG). Für die Insolvenz einer e.G. gilt die Besonderheit, dass zwar aus Gründen der Überschuldung ein Insolvenzverfahren gegen sie eröffnet werden kann, dies aber nach $\int 98$ GenG nur dann möglich ist, wenn

- die Genossen Nachschüsse zu leisten haben, und die Überschuldung ein Viertel des Gesamtbetrags der Haftsummen aller Genossen übersteigt,

- die Genossen keine Nachschüsse zu leisten haben, oder

- die Genossenschaft aufgelöst ist.

9 Nach $\$ 73$ GenG bestimmt sich die Auseinandersetzung mit dem ausgeschiedenen Mitglied nach der Vermögenslage der Genossenschaft („Auseinandersetzungsguthaben“) und dem Bestand der Mitglieder zur Zeit des Ausscheidens. 


\section{A.3.1.3. Der eingetragene Verein (e.V.)}

Sowohl für das zentralistische Modell wie für das dezentrale Modell hat TPo den eingetragenen Verein (e.V.) als mögliche Organisationsform verortet. Das spricht für die Flexibilität dieser Gesellschaftsstruktur, die sich für den kleinen Taubenzüchterverein ebenso eignet wie für den Deutschen Fußballbund mit Millionenumsätzen. Es muss allerdings betont werden, dass der nicht wirtschaftlich ausgerichtete und deshalb eintragungsfähige Verein primär keine Gewinnerzielung verfolgen darf, was jedoch nicht bedeutet, dass keine Einnahmen gemacht werden können. Für die Verbindlichkeiten eines e.V. haftet grundsätzlich nur das Vereinsvermögen, die persönliche Haftung der Mitglieder ist ausgeschlossen. Auf den Vorstand, der die laufenden Geschäfte führt oder an einen Geschäftsführer delegiert, kann allerdings bei mangelnder Aufsichtsführung durchaus durchgegriffen werden. Eine etwaige Ressortaufteilung schützt den Vorstand nach der einschlägigen Rechtsprechung nicht davor, in bestimmten Fällen persönlich in die Haftung genommen zu werden. Dies ist insbesondere dann der Fall, wenn Verletzungen öffentlich-rechtlicher Pflichten (Steuerpflichten des Vereins, Abführung von Sozialversicherungsbeiträgen) oder deliktische Verstöße (z.B. Insolvenzverschleppung oder ähnliches) im Raume stehen.

Die Autoren von TPo haben bereits hervorgehoben, dass die Verantwortlichkeit für die BMB im Falle von Meinungsverschiedenheiten innerhalb eines e.V. entweder beim Trägerorgan wie Vorstand bzw. Geschäftsführung liegt oder „beim Vertretungsorgan der übergeordneten Trägereinrichtung (Rektor/Kanzler der Universität, Klinikdirektor). In jedem Fall obliegt die Leitung der BMB einem Kollegialorgan“ (3.3. Ziffer 4). Die sich daraus möglicherweise ergebenden längeren Entscheidungswege könnten durch eine starke Geschäftsführung vermieden werden. Wegen seiner ideellen Zielsetzung, und da sich der e.V. als Rechtsform in der wissenschaftlichen Forschung bereits bewährt hat, genießt der Verein in der Regel ein großes öffentliches Vertrauen. Je nach Einbindung in universitäre oder klinische Gegebenheiten ist auch eine größere Langlebigkeit gegeben. Vorsorge für den Fall der Insolvenz oder der Auflösung kann in der Satzung getroffen werden. Vereine neigen in aller Regel wenig zu Umwandlungen, was in ihrem zumeist auf Gemeinnützigkeit ausgerichteten Zweck begründet liegt. Trotzdem sind Umwandlungen auch bei Vereinen prinzipiell möglich, z.B. in eine gemeinnützige $\mathrm{GmbH}$.

\section{A.3.1.4. Die Gesellschaft mit beschränkter Haftung (GmbH)}

Die GmbH ist als Rechtsform für alle drei in TPo formulierten Modellszenarien denkbar, da sie auch für nichtgewerbliche, sprich wissenschaftliche Zwecke gegründet werden kann. Im Bereich der wissenschaftlichen Forschung hat sich die $\mathrm{GmbH}$ bereits bewährt, etwa bei den früher so genannten „Blaue Liste“Instituten oder den Leibnitz-Einrichtungen. Die Gestaltung einer $\mathrm{GmbH}$ ist sehr flexibel, von voller Entscheidungsfreiheit der Gesellschafter bis hin zu weitgehenden Mitverwaltungsrechten der Gesellschafter durch das Organ der 
Gesellschafterversammlung. Als juristische Person ist die Fortführung einer $\mathrm{GmbH}$ auch bei Ausscheiden oder Auswechseln von Gesellschaftern grundsätzlich gewährleistet. Im Hinblick auf die weiter unten (E.I.3.I.) diskutierte Aufteilung einer BMB in Rechtsträger und Betriebsgesellschaft erscheint eine $\mathrm{GmbH}$ geeignet als Rechtsträgergesellschaft, wenn nicht aus Gründen der Reputation hierfür eine öffentlich-rechtliche Organisationsform oder eine Stiftung vorgezogen wird.

In der Regel ist der Kapitaleinsatz der Gesellschafter einer $\mathrm{GmbH}$ zunächst begrenzt, und nur mit diesem Kapital wird auch gehaftet. Die Praxis sieht aber oft anders aus, so dass sogar schon von einer Aushöhlung dieser Gesellschaftsform gesprochen wird. Banken und andere Geldgeber stellen zunehmend nur dann weiteres Kapital zur Finanzierung von GmbHs zur Verfügung, wenn sich die Gesellschafter persönlich in die Haftung nehmen lassen. In der Tat kommt es dann in vielen Fällen zur Gründung einer Mischform zwischen einer Personengesellschaft (mit stark persönlichem Haftungseinschlag der Gesellschafter) und einer Kapitalgesellschaft (mit entsprechenden Haftungsbeschränkungen). Langlebigkeit verbindet sich weniger mit der Rechtsform einer $\mathrm{GmbH}$ als mit anderen gesellschaftsrechtlichen Strukturen, selbst wenn durch entsprechende vertragliche Gestaltung Vorsorge gegen den Untergang oder Missbrauch der Probensammlungen getroffen werden kann. Finanzielle Unabhängigkeit ist zwar grundsätzlich gegeben, in der Praxis hängen die meisten GmbHs aber stark von ihren Kapitalgebern ab. Der Übergang in eine andere Gesellschaftsform ist ohne weiteres möglich. Die bei Gründung einer $\mathrm{GmbH}$ intendierte beschränkte Haftung kann sich unter Umständen negativ auf das Image einer BMB auswirken und der öffentlichen Vertrauensbildung entgegenstehen. Auf der anderen Seite stellt man sich unter einer $\mathrm{GmbH}$ vielfach eine schlagkräftige Organisationsform vor, die flexibel zu handeln in der Lage ist; ein Eindruck, der das Vertrauen in die Wettbewerbs- und Durchsetzungsfähigkeit einer BMB durchaus stärken könnte.

\section{A.3.1.5. Die Stiftung des privaten Rechts}

Die (gemeinnützige) Stiftung ist bisher wenig als mögliche Rechtsform für BMBs diskutiert worden und taucht auch in TPo nicht als praxisrelevante Organisationsform auf. ${ }^{10}$ Die Stiftung bietet hinsichtlich Vertrauensbildung, Langlebigkeit und Insolvenzsicherheit erhebliche Vorteile gegenüber anderen Rechtsformen. Zur Führung einer Stiftung kann ein (schlagkräftiger) Vorstand bestellt werden, der durch den (möglichen) Stiftungsrat überwacht wird. Stiftungen sind durch eine große finanzielle Unabhängigkeit charakterisiert. Allerdings muss das Stiftungskapital erst einmal eingeworben werden. Da nur das Stiftungsvermögen haftet, sind alle Mitwirkenden von der persönlichen Haftung ausgeschlossen. Alle Erträge werden dem Stiftungsvermögen zugeführt. Als Nachteil einer Stiftung werden gelegentlich lähmenden Meinungsverschiedenheiten zwischen den Selbstverwaltungsorganen angeführt. Außerdem gibt 
es keine Möglichkeit, den Stiftungszweck nachträglich zu ändern. Dieses Problem kann aber durch flexibel formulierte und vorausschauende Festlegungen in der Stiftungssatzung weitgehend entschärft werden. Wegen ihrer Ausrichtung auf Gemeinnützigkeit sind die Umwandlungsmöglichkeiten bei der Stiftung gering.

\section{A.3.2. Bewertung}

Die in den Abschnitten A.2. und A.3. vorgestellten Gesellschaftsformen sind zum Teil sehr flexibel gestaltbar und können der jeweiligen Interessenlage angepasst werden. Allerdings spricht im Kontext der BMB Vieles gegen den als Grundform der Personengesellschaft auch in die Betrachtung einbezogenen Einzelkaufmann. Interessanterweise entsprechen die im Rahmen von TPo in der Praxis vorgefundenen Gesellschaftsformen im Wesentlichen denjenigen, die aufgrund der hier angestellten rein rechtlichen Betrachtung am ehesten in Frage $\mathrm{zu}$ kommen scheinen. Insofern decken sich Evaluierung und Rechtsformuntersuchung. Dass die Stiftung in TPo nicht auftauchte, liegt wahrscheinlich daran, dass ein Stiftungszweck im Bewusstsein der Akteure und der Öffentlichkeit zunächst erst wachgerufen werden muss und die Stiftung deshalb eher in Zukunft eine Bedeutung im Kontext der BMBs haben wird.

\section{A.3.3. Rechtsformentableau}

\begin{tabular}{|c|c|c|c|c|c|c|}
\hline \multirow[b]{2}{*}{ Kriterium } & \multicolumn{6}{|c|}{ Rechtsform } \\
\hline & GbR & $\mathrm{GmbH}$ & e.v. & eG & Stiftung & öffentl.-rechtl. \\
\hline $\begin{array}{l}\text { Vermeidung langwieriger } \\
\text { Entscheidungsprozesse }\end{array}$ & $x$ & $x$ & - & - & - & - \\
\hline Vertrauensbildung & $(X)$ & $(X)$ & $x$ & $x$ & $x$ & $x$ \\
\hline Langlebigkeit & - & $(X)$ & - & $x$ & $x$ & $x$ \\
\hline Insolvenzsicherheit & - & - & - & - & $x$ & $x$ \\
\hline Finanzielle Unabhängigkeit & $x$ & $x$ & $x$ & $x$ & $(X)$ & - \\
\hline $\begin{array}{l}\text { persönlicher Haftungsaus- } \\
\text { schluss }\end{array}$ & - & $x$ & $x$ & $x$ & $x$ & $x$ \\
\hline Umwandlungsmöglichkeit & $x$ & $x$ & - & - & - & - \\
\hline Gewinnerzielung & $x$ & $x$ & $(X)$ & $(X)$ & $(X)$ & $(X)$ \\
\hline Eignung „Zentralistisch“ & $x$ & - & - & - & - & $x$ \\
\hline Eignung „Dezentral“ & - & $x$ & $x$ & - & $x$ & $x$ \\
\hline Eignung „Kooperativ“ & - & - & - & $x$ & $x$ & - \\
\hline
\end{tabular}

$X$ : gegeben; $(X)$ : nur mit Einschränkung gegeben 


\section{A.3.4. Erläuterungen zum Rechtsformentableau}

Im voran stehenden Tableau wurden den verschiedenen Rechtsformen die Eigenschaften zugeordnet, die nach genereller Einschätzung für den Betrieb einer BMB notwendig oder wünschenswert sind.

\section{A.3.4.1. Vermeidung langwieriger Entscheidungsprozesse}

Größere Flexibilität ist grundsätzlich bei den mehr kapitalgesellschaftsrechtlich organisierten Unternehmen gegeben, wenn deren Satzung dies bestimmt. Die Gesellschafter können die Gesellschaftssatzung in diesem Sinne auch wesentlich beeinflussen. Natürlich hätte der Einzelunternehmer die größtmögliche Entscheidungsfreiheit, er wurde aber aus den oben genannten Gründen hier nicht berücksichtigt. Außerdem kann auch eine Ein-Personen-GmbH wie eine Einzelunternehmung geführt werden.

\section{A.3.4.2. Vertrauensbildung}

Das Vertrauen der Spender in eine BMB wird wesentlich davon abhängen, wie sicher Proben und Daten sind und ob sie ausschließlich für die vereinbarten Ziele eingesetzt werden. Insoweit scheint eine staatlich organisierte BMB den Bedürfnissen der Spender am besten gerecht zu werden. Allerdings sind auch Beschlagnahmen bei einer staatlich organisierten BMB am leichtesten durchführbar. Wenn man davon ausgeht, dass Spender ein Interesse daran haben, dass Forschungsergebnisse aus ihren Proben und Daten der Allgemeinheit zugute kommen oder sie selbst kontrollieren möchten, wem sie zugute kommen, dürfte eine Stiftung oder ein Probandenkollektiv, z.B. als e.G., diesen Interessen wohl am ehesten gerecht werden. Das Vertrauen der Öffentlichkeit in ein Unternehmen steigt zudem grundsätzlich auch mit dem Haftungskapital. In diesem Zusammenhang wäre zu bedenken, dass eine mit dem vorgeschriebenen Mindeststammkapital gegründete $\mathrm{GmbH}$ in der Regel nicht einmal einen Bankkredit ohne persönlich haftenden Bürgen (Gesellschafter) erhält.

\section{A.3.4.3. Langlebigkeit}

Kapitalgesellschaften haben den Vorteil, dass ihr Bestehen unabhängig von Inhaber- oder Mitgliederwechseln ist, was ihnen per se eine größere Langlebigkeit verleiht. Als besonders langlebig gelten die von gewissermaßen übergeordneten Interessen an ihrer Erhaltung geleiteten Genossenschaften und Stiftungen.

\section{A.3.4.4. Insolvenzsicherheit}

Im Falle einer Insolvenz wird für ein Unternehmen regelmäßig ein Insolvenzverwalter, zumeist ein Jurist oder Betriebswirt, eingesetzt. Diesem sind die zu beachtenden rechtlichen Vorgaben in Abhängigkeit des von ihm in der Insol- 
venz zu führenden Unternehmens bekannt oder er wird sich damit vertraut machen. Insolvenzverwalter werden von den zuständigen Insolvenzgerichten nicht zufällig, sondern nach ihren auf den jeweiligen Insolvenzfall bezogenen besonderen Kenntnissen und Fertigkeiten ausgesucht und bestellt. Bei Stiftungen und öffentlich-rechtlichen Trägern ist eine besonders hohe Insolvenzsicherheit als systembedingt anzunehmen, weil diese Einrichtungen vor und während ihrer Tätigkeit besonderer behördlicher Überwachung unterliegen. ${ }^{11}$

\section{A.3.4.5. Finanzielle Unabhängigkeit}

Bei Kapitalgesellschaften und juristischen Personen ist das Vermögen als Sondervermögen zu behandeln. Für die laufenden Geschäfte sind bei diesen Rechtsformen bestimmte Organe, Geschäftsführer oder Vorstände, einzusetzen, die über die Verwendung der zur Verfügung stehenden Finanzmittel zu befinden haben. Insofern liegt bei nicht streng Inhaber bezogenen Unternehmungen die größte finanzielle Unabhängigkeit. Je weniger die Kapitalgeber in das Tagesgeschäft einer Unternehmung involviert sind, desto freier darf sich das Leitungsorgan in der Regel bei der Mittelverwendung fühlen. In einer kleinen GmbH mit großer Nähe zu den Gesellschaftern kann die finanzielle Unabhängigkeit demgegenüber sehr eingeschränkt sein. Bei Vereinen und Stiftungen können zu eng gefasste Zweckbindungen in der Satzung zu Einschränkungen der finanziellen Unabhängigkeit führen.

\section{A.3.4.6. Persönlicher Haftungsausschluss}

Der Ausschluss der persönlichen Haftung des Forschers ist bei Kapitalgesellschaften oder juristischen Personen evident. Zu beachten ist aber, dass in den Fällen vorsätzlicher oder grob fahrlässiger Schäden neben der Haftung der Unternehmung auch eine persönliche Haftung des verantwortlichen Mitarbeiters eintreten kann. Das gleiche gilt, wenn Forscher zugleich als Geschäftsführer oder Vorstand tätig sind und in dieser Eigenschaft einen Schaden verursachen. Dann trifft sie neben der Haftung der Unternehmung eventuell eine Haftungsverpflichtung aus Organ- oder Organisationsverschulden. Letzteres tritt ein, wenn der Schaden durch einen bei der Unternehmung angestellten Arbeitnehmer verursacht wurde und dieser Schaden durch entsprechende innerbetriebliche Organisationsmaßnahmen oder hinreichende Aufsichtsführung zu vermeiden gewesen wäre.

\section{A.3.4.7. Umwandlungsmöglichkeit}

Die Umwandlung von einer Personengesellschaft in eine Kapitalgesellschaft oder juristische Person und umgekehrt ist relativ einfach und ist im Umwandlungsgesetz und dem AktG geregelt. Vereine, Genossenschaften und Stiftungen 
Teil A: Trägerschaft von BMB und geeignete Rechtsformen

neigen in aller Regel weniger zu Umwandlungen, was in ihrem zumeist auf Gemeinnützigkeit ausgerichteten Zweck begründet liegt. Trotzdem sind Umwandlungen auch bei diesen Rechtsformen prinzipiell möglich, so z.B. eines e.V. in eine gemeinnützige $\mathrm{GmbH}$.

\section{A.3.4.8. Gewinnerzielung}

Gewinnerzielung steht beim nicht wirtschaftlichen e.V., der e.G. und der Stiftung, aber auch bei der gemeinnützigen $\mathrm{GmbH}$ (gGmbH) nicht im Vordergrund. Wenn überhaupt, so sind erzielte Erträge an die Mitglieder auszuschütten bzw. verbleiben beim Stiftungsvermögen. Öffentlich-rechtliche Träger können sich über Gebühren finanzieren und auch Gewinne machen.

\section{A.3.4.9. Eignung einzelner Organisationsmodelle für eine BMB}

Für das zentralistische Modell einer BMB kommen typischerweise die stark auf den Unternehmer bezogenen Rechtsformen in Frage. Die dezentrale Organisationsform ist ohne weiteres bei Kapitalgesellschaften möglich, doch auch für Genossenschaften und Stiftungen ist unter Führung eines Zentralverbandes eine Organisation mit selbständigen Niederlassungen denkbar. Die bereits in Kooperation befindlichen BMB stellen einen Mix aus öffentlich-rechtlichen Trägern, e.V. und GmbH dar. Kooperativ, d.h. mit rechtlich jeweils selbständig geführten und in einer Kooperation zusammen geschlossenen Organisationsform, sind Kombinationen sämtlicher Rechtsformen möglich.

\section{A.4. Welcher spezifische Regelungsbedarf für den Aufbau und Betrieb einer BMB besteht in Abhängigkeit von der gewählten Rechtsform?}

Unter „Regelungsbedarf“ versteht man im juristischen Sprachgebrauch den an den Gesetzgeber zu formulierenden Bedarf auf Einführung einer neuen oder veränderten Gesellschaftsform. Im Kontext der BMB könnte dies der Wunsch nach Neugestaltung von Rechtsformen für den spezifischen Bedarf von BMB sein. Einer solchen Anforderung, würde sie denn gestellt, würde mit Sicherheit nicht in absehbarer Zeit stattgegeben. Soweit ersichtlich, besteht aber wegen der Vielgestaltigkeit der vorhandenen Unternehmensformen kein spezifischer gesetzlicher Regelungsbedarf, zumal die in den verschiedenen Gesetzen geregelten Gesellschaftsformen nur Grundgestaltungen sind, die für den jeweiligen Bedarfsfall zum Teil weitgehend umgestaltet werden können. Davon zu unterscheiden sind bestimmte sinnvolle Einzelerfordernisse wie die Registrierung von BMB, die Einführung einer Treuhandschaft oder zusätzliche Unternehmenskontrollen, die unabhängig von der gesellschaftsrechtlichen Gestaltung sind. 
A.4. Welcher spezifische Regelungsbedarf für den Aufbau und Betrieb

einer BMB besteht in Abhängigkeit von der gewählten Rechtsform?

Regelungen, die Organisationsvorgaben, innerbetriebliche Weisungswerke oder Allgemeine Geschäftsbedingungen und ihre notwendigen oder zweckmäßigen Inhalte zum Gegenstand haben, werden im Fortgang des Biobanken-Projekts noch zu entwickeln sein. ${ }^{12}$ Dies gilt zum einen für das mit einer Insolvenz verbundene Problem des Fehlgebrauchs der dort gelagerten Proben und Daten. Hierfür kann derzeit durch interne Regelungen, etwa im Rahmen der Satzung, Vorsorge getroffen werden. Auf der Ebene der Rechtsformen ließe sich dem Problem durch die Wahl einer entsprechenden Trägerschaft begegnen (öffentlich-rechtlich, Stiftung). Wird eine BMB tatsächlich aufgelöst, so stellt sich die Frage, was mit dem zugehörigen Probenbestand passieren soll. Derzeit kann man davon ausgehen, dass alle Proben und Daten vernichtet werden müssen. Für die Zukunft ließe sich dieser Verlust wertvoller wissenschaftlicher Ressourcen dadurch vermeiden, dass der Gesetzgeber eine rechtliche Regelung schafft, wonach die BMB bzw. die bei ihr vorhanden Proben und Daten einem zu bestimmenden und (auf Bundesebene) noch zu schaffenden zentralen Träger angeboten werden müssten.

In der Satzung oder im Gesellschaftsvertrag der BMB sind die jeweiligen Geschäftsführungszuständigkeiten zu regeln. Hier könnte an eine Unterscheidung zwischen allgemeiner und besonderer Geschäftsführung für spezielle Problemkreise bzgl. Datenschutz und BMB-Materialien bzw. Forschung gedacht werden. In Verträgen mit an der BMB forschenden Wissenschaftlern sind alle Verwertungs-, Geheimhaltungs- bzw. Veröffentlichungsregelungen zu treffen und Fragen des Namensnennungsrechts zu klären. In Verträgen mit Zulieferern der BMB muss eine Freistellungs- und Prozessunterstützungsklausel bezüglich der Haftung im Falle der Rechtsverletzung eines Spenders enthalten sein. Der Zulieferer sollte ferner durch eine entsprechende Erklärung versichern, dass er Rechtsinhaber ist bzw. erklären, welche Rechte er innehat, dass Rechte Dritter nicht betroffen sind und dass er rechtmäßig in den Besitz der Materialien gekommen ist. Ferner sollte ein Muster der von den Spendern verlangten Einwilligungserklärung als Anlage zum Vertrag beigefügt werden. 